\title{
A New Method to Measure Parameters of Frequency-Selective Radio Channels Using Power Measurements
}

\author{
Klaus Witrisal, Student Member, IEEE, Yong-Ho Kim, and Ramjee Prasad, Senior Member, IEEE
}

\begin{abstract}
This paper describes a method of deriving channel parameters for a time-dispersive (=frequency-selective) radio channel from simple wide-band power measurements. A novel relationship is found allowing the estimation of the root-mean-square (rms) delay spread from such data. Furthermore, we describe a set of equations that can be used for fitting the (measured) channel parameters to a mathematical model, the so-called frequency-domain (FD) model. We also show a simulation procedure, which directly implements the mathematical description of the channel. The output of this procedure-realizations of frequency-selective channel transfer functions-may be used for instance in the investigation of OFDM systems. The study is restricted to the small-scale modeling; Rayleigh and Ricean fading channels are considered. The estimation of the rms delay spread is based on the frequency-domain level crossing rate $\left(L_{C} R_{f}\right)$, which is derived from the FD-channel model. It is shown that the rms delay spread is proportional to the $L_{C R}$. Because of the simple hardware required for finding the $L_{C} R_{f}$, the suggested measurement method is particularly interesting for the millimeter-wave frequency band (>30 GHz). However, it can be used at other frequencies as well, where standard laboratory equipment is sufficient for conducting the measurements. The accuracy of this technique depends on the bandwidth observed and can be increased further by combining multiple measurements performed within a small local area.
\end{abstract}

Index Terms-Average bandwidth of fades, channel measurement, channel modeling, frequency domain modeling, frequency-selective fading, level-crossing rate, Rayleigh channels, Ricean channels, rms delay spread.

\section{INTRODUCTION}

$\mathbf{R}$ ADIO PROPAGATION in a mobile radio channel is determined mainly by multipath interference, called smallscale fading. As an effect of it, the narrowband-received power fluctuates dramatically when observed as a function of location (or time) and frequency. In the early days of mobile systems, the communications engineer was mainly interested in

Paper approved by R. A. Valenzuela, the Editor for Transmission Systems of the IEEE Communications Society. Manuscript received January 15, 1999; revised March 15, 2000 and September 11, 2000. This work was conducted under a cooperative research program between the Access Network Lab, Korea Telecom, Korea, and the Centre for Wireless Personal Communications (CEWPC), Delft University of Technology, The Netherlands. This paper was presented in part at the IEEE PIMRC Symposium, Boston, MA, Sept. 1998.

K. Witrisal is with the Department of Electrical Engineering, Delft University of Technology, NL-2628 CD Delft, The Netherlands (e-mail: K.Witrisal@ITS.TUDelft.NL).

Y.-H. Kim is with the Access Network Laboratory, Korea Telecom, Seoul 137-792, Korea (e-mail: yhokim@kt.co.kr).

R. Prasad is with the Center for PersonKommunikation, Aalborg University, DK-9220 Aalborg, Denmark (e-mail: prasad@cpk.auc.dk).

Publisher Item Identifier S 0090-6778(01)09100-0. the time-variability of narrowband channels, which were thus studied extensively (see, e.g., [1]). By that time, transmission bandwidths were small, thus flat-fading was a reasonable assumption. As the systems evolved, demand for higher transmission rates has been increasing, making the channel's time dispersion (=frequency-selectivity) a major issue in the design of air-interfaces for digital communications. Currently, the wireless extension of existing local area networks (WLAN) is being researched by many groups. Most of the proposals use orthogonal frequency division multiplexing (OFDM) as a modulation scheme [2] and assume a static channel during the transmission of one OFDM symbol. In such systems, many of the methods developed for narrow-band, single-carrier transmission can be employed in the frequency-domain. This was the main motivation for this study.

The so-called frequency-domain channel model (FD-model) is proposed and analyzed in this paper. It characterizes the frequency-selective fading by the spaced-frequency correlation function of the channel. To be specific, the spaced-frequency correlation function is defined by its Fourier transform, the delay power spectrum (DPS) [3]. This approach is dual to defining the time-variability by the Doppler power spectrum, which is widely known as Jakes' fading model [1].

Using the FD-model, which is a description of the DPS specified by just two to four parameters, an elaborate mathematical analysis of the frequency-selective fading is performed. Expressions are given, relating the model parameters to the most important channel parameters, like normalized received power (NRP), ${ }^{1}$ Ricean K-factor, and root-mean-square (rms) delay spread (RDS). The level crossing rate (LCR) and the average duration of fades (ADF) are interesting parameters in the design of transmission systems over time-variant channels. In this paper, the LCR in the frequency-domain $\left(\mathrm{LCR}_{\mathrm{f}}\right)$ and the average bandwidth of fades (ABF) are derived, which provide comparable information for the design of OFDM based systems. They also allow interesting conclusions on the relevance of the channel parameters listed above for specifying this type of channels.

Based on the proposed FD-model it is shown that the $\mathrm{LCR}_{\mathrm{f}}$ is proportional to the RDS. A property that can be used for estimating the RDS from noncoherent channel measurements [4]. Therefore, full sets of channel parameters (NRP, $K$ factor, RDS) can be derived from the measured magnitudes only of

\footnotetext{
${ }^{1}$ The (dimensionless) normalized received power is defined as the ratio of the received power $P_{r}$ and the transmitted power $P_{t}$. Equivalently, the absolute received power $P_{r}[\mathrm{~W}]$ could be used for channel characterization.
} 
channel transfer functions (TF). Standard laboratory equipment may be used to conduct such measurements, as e.g., a swept-frequency continuous-wave $(\mathrm{CW})$ signal generator and a power meter or spectrum analyzer. Its simplicity makes the method particularly useful at extremely high frequencies $(>30 \mathrm{GHz}$; millimeter wave band), where for instance network analyzers become very cumbersome and expensive.

Since the measurement method proposed in this paper is based on a statistical model, a sufficiently large bandwidth must be observed to obtain high accuracy, just like a large frequency-band must be scanned with a network-analyzer, in order to obtain a certain time-resolution. An advantage of our method is that-because of ergodicity - the observation bandwidth can be increased by analyzing the combined data of a number of "narrow-band" measurements performed in a "local area." The size of this local area must be selected sufficiently small for the channel parameters not to vary due to shadowing. Well de-correlated spectra can be obtained when the receiver's location is changed in the order of the wavelength $\lambda$. Especially at the millimeter wave band with wavelengths below $1 \mathrm{~cm}$, many spectrum samples can be taken within small areas.

Note that due to the lack of phase information, the Fourier transform cannot be used for transforming a magnitude TF to the delay time-domain, which would allow determining delay spread parameters as the RDS. However, the causality of the impulse response implies that the Hilbert transform describes the relationship between the real and imaginary components of the complex valued TF. Donaldson et al. have applied this property for analyzing magnitude TFs [5], yielding estimates of the channels' impulse responses. Their method can be an alternative way of determining delay spread parameters using this type of measurements.

The paper is organized as follows. The FD-channel model is introduced in Section II. A simulation scheme for time-invariant channels is presented in Section III; its extension to the time-variant case is discussed. In Section IV, the mathematical analysis of the frequency selectivity is given, based on the model. Using the $\mathrm{LCR}_{\mathrm{f}}$, the RDS can be estimated from noncoherent channel measurements; these investigations are described in Section V. Finally, conclusions and recommendations are given in Section VI.

\section{Frequency-Domain Channel Modeling}

\section{A. Channel Characterization}

This work is based on a mathematical description of the mobile radio channel as a linear time-variant system. Specifically, the time-variant transfer function $H(f, t)$ is considered from the family of Bello's system functions, defined as [6], [7]

$$
z(t)=\int_{-\infty}^{\infty} H(f, t) U(f) e^{j 2 \pi f t} d f
$$

$U(f)$ is the input spectrum of the channel and $z(t)$ is the output signal, both in complex lowpass equivalent notation. $H(f, t)$ is the complex envelope of the received signal for a complex exponential input at frequency $f$.

To simplify the mathematical analysis, we assume to have a wide-sense stationary and uncorrelated scattering (WSSUS)

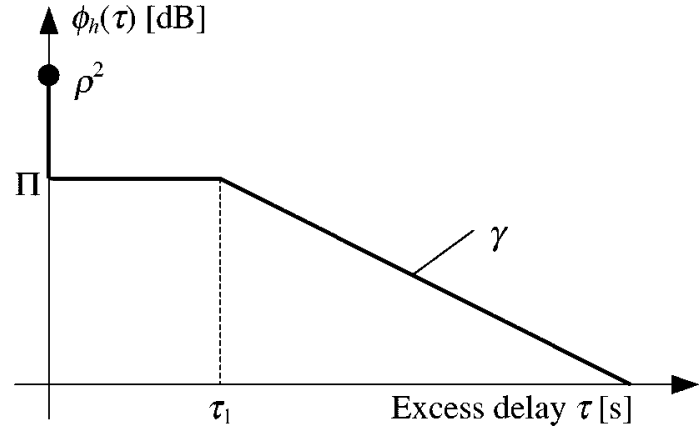

Fig. 1. Model of the DPS.

channel [6], [7], [3]. This implies for the time-variant transfer function $H(f, t)$ to be wide-sense stationary (WSS) with respect to both variables $f$ and $t$. The channel is thus characterized for all time and all frequencies by the two-dimensional spaced-frequency, spaced-time correlation function ${ }^{2}$

$$
\phi_{H}(\Delta f, \Delta t)=E\left\{H^{*}(f, t) H(f+\Delta f, t+\Delta t)\right\} .
$$

Rayleigh and Ricean fading channels are described by assuming respectively a zero or nonzero mean complex Gaussian process for the first order statistics of $H(f, t)$.

Most of the analysis presented in the rest of the paper concentrates on the case of the time-invariant frequency-selective channel. The channel is then described by the TF $H(f)$, which is a WSS complex Gaussian stochastic process in $f$, according to the above assumptions. The second order statistical functions characterizing $H(f)$ are the spaced-frequency correlation function $\phi_{H}(\Delta f)=\phi_{H}(\Delta f, 0)$ and its Fourier transform (FT), the delay power spectrum (DPS) $\phi_{h}(\tau)$, where $\tau$ is the channel's (excess) delay time. Per definition, the first (direct) path has a delay time of $\tau=0$. A mathematical description of the DPS will be the basis of the analysis presented in this paper. We call this model the FD-channel model (see Section II-B).

More familiar is the dual approach of modeling the time variability of a narrowband channel as a WSS complex Gaussian stochastic process $H(t)$. An example for this method is widely known in the literature as Jakes' fading model [1]. Compared to the FD model, the frequency variable is exchanged with the time variable, and the second order statistics are the spaced-time correlation function $\phi_{H}(\Delta t)=\phi_{H}(0, \Delta t)$ and the Doppler power spectrum $S_{H}(\nu)$, which are a Fourier pair as well. $\nu$ is the Doppler frequency.

\section{B. Modeling of the Time-Invariant Wide-Band Channel}

In agreement with measurements reported in [8], the shape of the DPS is defined as shown in Fig. 1. It is characterized by four parameters: $\rho^{2}$ - the normalized power of the direct ray; $\Pi$ $[1 / \mathrm{s}]$ - the normalized power density of the constant-level part; $\tau_{1}[\mathrm{~s}]$ — the duration of the constant level part; and $\gamma[1 / \mathrm{s}]$ - the

\footnotetext{
${ }^{2}$ Bello [6] has also introduced the quasi-WSSUS channel (QWSSUS) in order to apply the concept of the WSSUS channel to real radio channels. A QWSSUS channel has the properties of a WSSUS channel for a limited bandwidth and for limited time or within a limited area. The dimensions of such an area (often called "local area" in connection with channel measurements) are in the order of $5 \ldots 40 \lambda$, where $\lambda$ is the wavelength.
} 


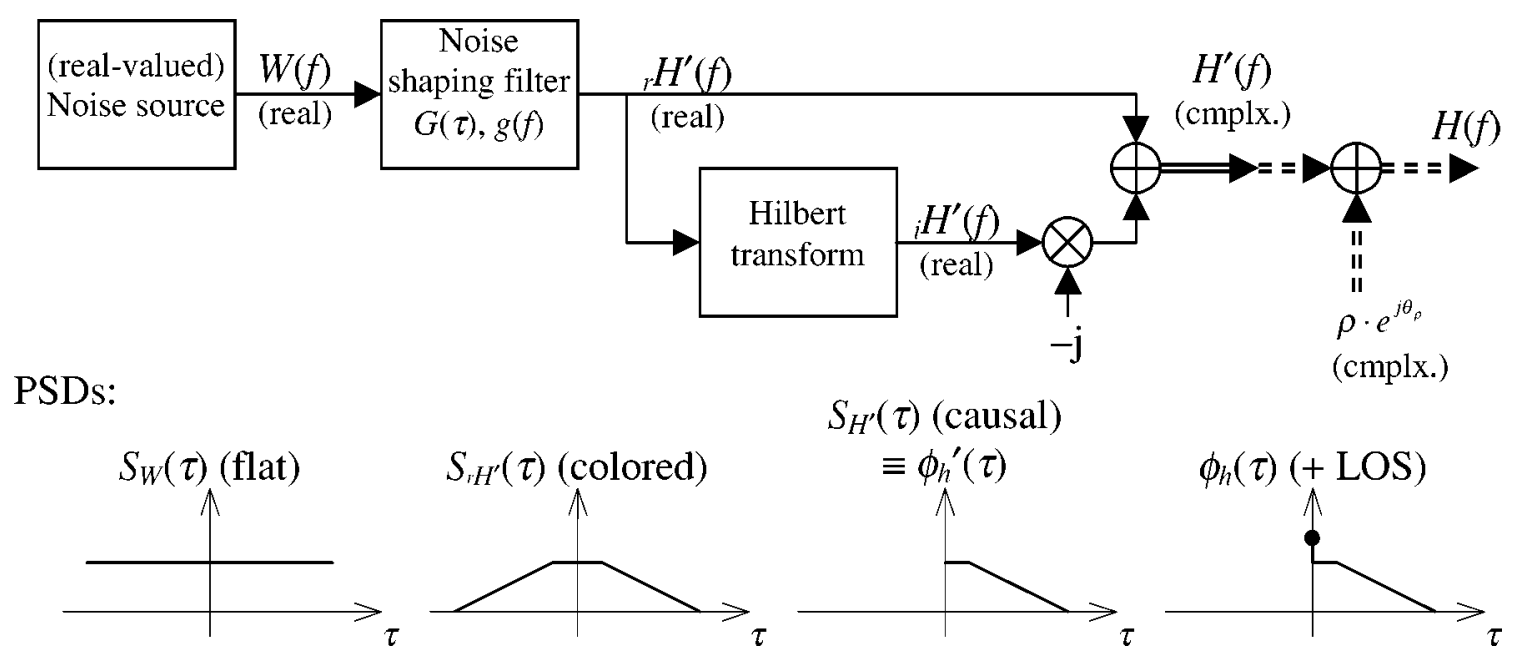

Fig. 2. Frequency-domain simulation of the frequency-selective radio channel.

decay exponent of the exponentially decaying part. Mathematically, the DPS can be written as

$$
\phi_{h}(\tau)= \begin{cases}0 & \tau<0 \\ \rho^{2} \delta(\tau) & \tau=0 \\ \Pi & 0<\tau \leq \tau_{1} \\ \prod e^{-\gamma\left(\tau-\tau_{1}\right)} & \tau>\tau_{1} .\end{cases}
$$

In many cases, the number of (free) parameters can be further decreased. The exponentially decaying DPS is a good approximation for most practical channels, which is implemented by letting $\tau_{1}=0$. The existence of a line-of-sight (LOS) ray at $\tau=0$ implies that the channel TF has nonzero mean, thus the fading envelope distribution is Ricean. Rayleigh fading channels have $\rho=0$.

For the analysis it is appropriate to define $u=\tau_{1} \gamma$, being a single parameter to account for the shape of the DPS. $u$ can take values $u \in[0, \infty]$, where the two extreme cases $u=0$ and $u=\infty$ define an exponentially decaying and a rectangular DPS, respectively. Note that in the latter case (rectangular DPS), the maximum excess delay will be much smaller than for $u=0$, thus $u$ can be used to adjust this parameter. Relations between the model parameters defined above and the channel parameters are presented in Section IV.

\section{Validity of the Model}

The proposed model is most appropriate for indoor propagation channels at limited ranges, especially when the transmitter and receiver are located within the same room. (The latter is a necessity for mm-wave communications.) In this case, all the impinging rays arrive within a compact "cluster" of impulses, which is well described by the DPS shown in Fig. 1 (see, e.g., [8], [9]). Due to first reflections arriving at similar strength, a constant-level part is sometimes observed. However, this depends on the antennas' radiation patterns [8]. Section III-C shows the validation of the model for such environments, using measurement results.

Clustering of rays is a property typical to indoor channels at longer ranges and at lower carrier frequencies, where propaga- tion through walls is possible [10]. This could be implemented in the proposed model by modifying the DPS accordingly. Similarly, arbitrary outdoor channels could be realized. However, for the mathematical analysis presented in this paper, we confine ourselves to the simple model shown above, with its small number of free parameters.

Since the proposed measurement method is based on this DPS-model as well, the impact of the actual channel model on the measurement results is studied in Sections IV-C and IV-D.

\section{Simulation OF FREQUENCY-SELECtive ChanNELS}

In this paper, the discussion of simulation schemes is mainly restricted to the case of static (time-invariant) frequency-selective channels. Such simulations are-for instance-appropriate for the study of OFDM systems, with a system model that reduces the channel including the IFFT at the OFDM transmitter and the FFT at the receiver to a set of parallel Gaussian (sub-) channels [11]-[13]. These sub-channels have complex attenuation factors given by the channel's TF $H(f)$ at the frequency instants of the OFDM sub-carriers. The simulation scheme presented in this section directly generates $H(f)$ for well-defined channel parameters.

\section{A. Model Description}

The simulation system for time-invariant channels is shown in Fig. 2. Independent FD-samples $W(f)$ of the frequency response are generated by a real-valued, Gaussian noise source. The appropriate spaced-frequency correlation is obtained by FD-filtering of $W(f)$ with a (low-pass) filter $g(f)$. The output of this filter is the real-valued, colored noise process ${ }_{r} H^{\prime}(f)=W(f) * g(f)$, where $*$ denotes convolution. The inverse FT of ${ }_{r} H^{\prime}(f)$ (in delay-time-domain representation) is complex valued and hermitian, i.e., symmetric with respect to the $\tau=0$ axis. It is not causal, in contrast to the impulse response (IR) of a real channel. The required causality in the time-domain is obtained by applying the Hilbert transform to ${ }_{r} H^{\prime}(f)$ and adding the result ${ }_{i} H^{\prime}(f)$ as $H^{\prime}(f)={ }_{r} H^{\prime}(f)-j{ }_{i} H^{\prime}(f)$. Doing this, the negative part of the IR is canceled. 

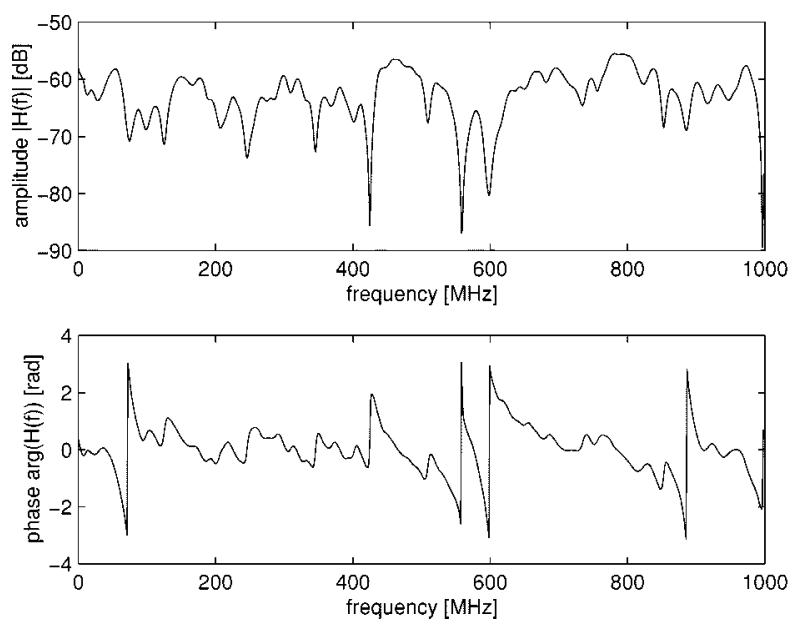

(a)

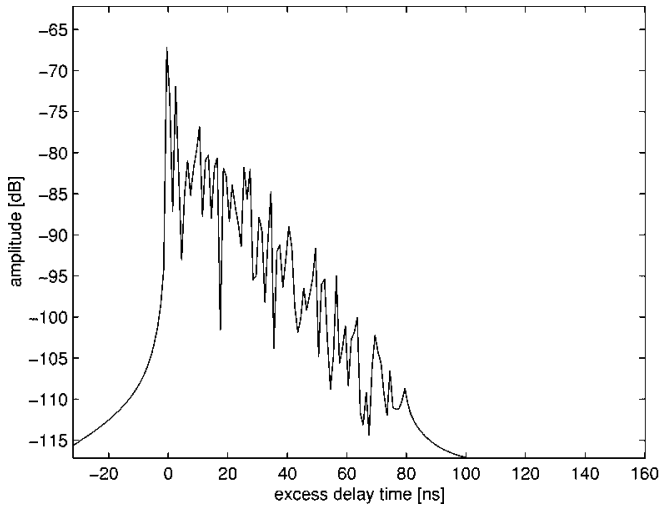

(c)
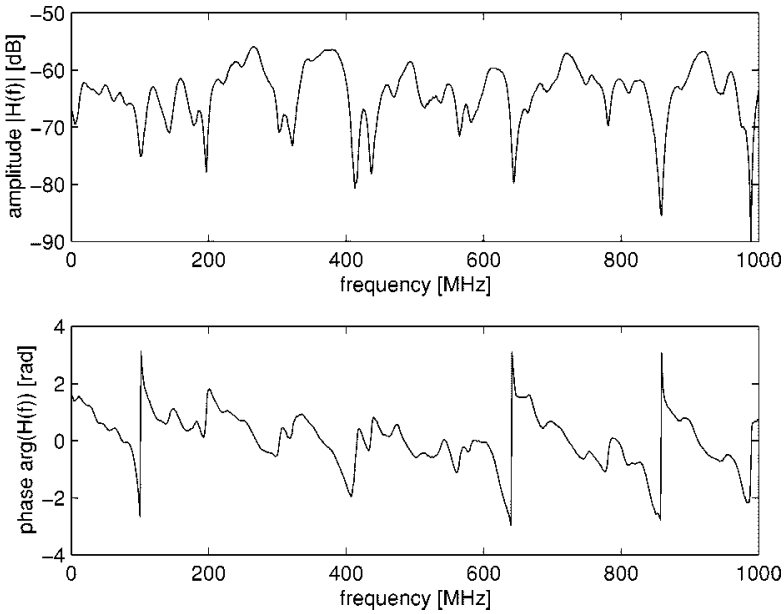

(b)

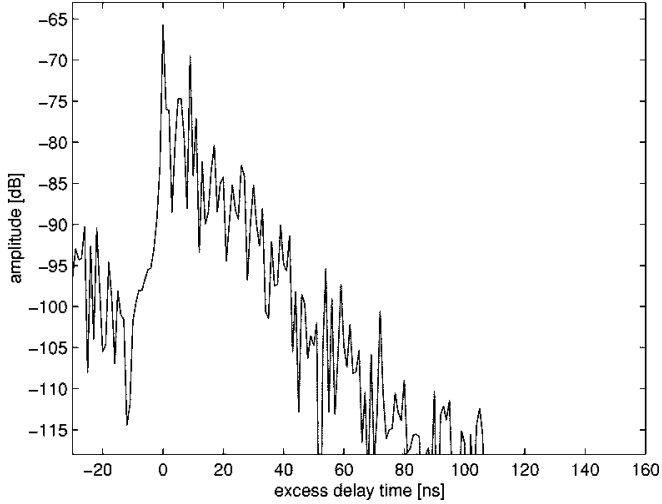

(d)

Fig. 3 (a) Simulated transfer function (TF). (b) TF measured with a network analyzer (corrected for linear phase shift). (c) and (d) Impulse responses derived by IDFT from the TFs (a) and (b), respectively.

The amplitude of the TF $\left|H^{\prime}(f)\right|$ is Rayleigh distributed since $H^{\prime}(f)$ is a complex Gaussian noise process. A Ricean fading channel may be simulated by adding a complex constant $\rho \cdot e^{j \theta_{\rho}}$ to $H^{\prime}(f)$, representing the LOS path at $\tau=0$.

\section{B. Implementation of the Simulation Scheme}

To obtain a computer simulation program producing TFs with the desired DPS, two elements of the above simulation scheme must be appropriately designed; the noise-shaping filter $g(f)$ and the variance of the noise source $\sigma_{W}^{2}$. The simulator produces a sampled version of the TF, $H\left(n f_{s}\right)$, where $f_{s}[\mathrm{~Hz}]$ is the sampling interval ( $n=\{0,1, \ldots, N-1\})$. $f_{s}$ must be selected according to the sampling theorem, i.e., $f_{s}<1 / 2 T_{m}$, where $T_{m}$ is the maximum excess delay of the channel's IR.

The power spectral density (PSD) of the output of the simulation scheme shown in Fig. 2 has to match the continuous DPS defined by (3). This is obtained by defining the filter $g(f)$ to have a TF $G(\tau)$ proportional to the DPS (for $\tau>0$, i.e., skipping the LOS component). Any classic filter design method can be used in this process [14]. By definition we let $|G(\tau)|=1$ during the constant-level part (or at $\tau=0^{+}$-if there is no constant-level part), which leads to the variance $\sigma_{W}^{2}=\Pi /\left(4 f_{s}\right)$, as derived in Appendix A.

In order to extend this static simulation scheme to a time-variant one, the $\mathrm{TF} H\left(f_{c}, t\right)$ must have the required
Doppler spectrum when the time-variations are investigated at any particular frequency $\left(f=f_{c}\right)$. This may be achieved by generating a number of independent TFs $H(f, t=m T)$, $m=\{1,2,3, \ldots\}$ and filtering them in time-direction at each frequency sample, according to a specific Doppler spectrum. [Separability of the joint time-frequency correlation function $\phi_{H}(\Delta f, \Delta t)$ is assumed $\left.{ }^{3}.\right]$

It should be noted at this point that the time-variant simulation scheme is very complex. It might thus be preferable to use a conventional fading simulator-one that generates a (time-variant) IR - and transform the IR to the frequency-domain, if required.

\section{FD-Simulation Results}

In Fig. 3, a simulated TF is shown [Fig. 3(a)] and compared to a measured one [Fig. 3(b)]. The two channels' IRs are given in Fig. 3(c) and (d), both derived from the respective TFs using the inverse discrete FT (IDFT) without windowing. The measurement was performed with a network analyzer, observing a bandwidth of $1 \mathrm{GHz}$ around a center frequency of $11.5 \mathrm{GHz} .^{4}$

${ }^{3}$ Separability of the two-dimensional spaced-frequency, spaced-time correlation function $\phi_{H}(\Delta f, \Delta t)$ means that it can be written as a product $\phi_{H}(\Delta f, \Delta t)=\phi_{H}(\Delta f) \phi_{H}(\Delta t)$. This assumption is valid if $T_{m} B_{d} \ll 1$ [15], which is given for practical propagation channels $\left(T_{m}\right.$ denotes the maximum excess delay; $B_{d}$ is the Doppler spread). 

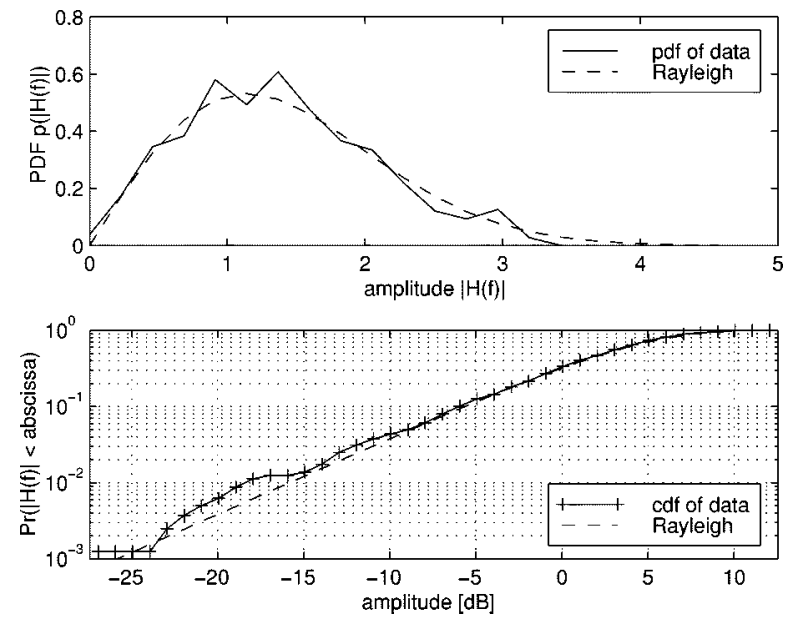

Fig. 4. pdf and cdf of the amplitude of the simulated transfer function.

Using the method explained in Section V, the channel parameters (NRP: $P_{0}=-62.1 \mathrm{~dB}, K=-1.9 \mathrm{~dB}$, RDS: $\tau_{\text {rms }}=$ $9.0 \mathrm{~ns}$ ) were extracted from the amplitude of the measured TF [Fig. 3(b)]. These parameters and $\tau_{1}=0$ were used to generate the simulated TF shown in Fig. 3(a). As the measured TF, it has a length of 801 samples. A 15-tap FIR filter was used for the noise shaping filter $g(f)$ in the simulation scheme.

The TF is obtained from a stochastic simulation model. Therefore we do not expect it to be identical to the measured one. However, it is clearly seen that the characteristic of the fading is well reproduced. Originally, a linearly increasing phase shift was evident in the measured TF corresponding to the propagation delay of the shortest path. In the illustration this was compensated for, to have the first component arrive at (excess) delay $\tau=0$.

Probability density function (pdf) and cumulative distribution function (cdf) of the simulated amplitude TF are shown in Fig. 4 and compared to the Rayleigh distribution. Because of the low $\mathrm{K}$-factor $(K=-1.9 \mathrm{~dB})$, good agreement is evident.

Second order statistical properties estimated from simulated TFs are shown in Fig. 5. The power spectrum obtained by averaging over periodograms of 100 simulated TFs agrees well with the used DPS model (upper plot). The lower plot shows (spaced-frequency) correlation properties and compares them to the theoretical function, which is derived from the DPS via the Fourier transform

$$
\begin{aligned}
\phi_{H}(\Delta f)= & F\left\{\phi_{h}(\tau)\right\} \\
= & \rho^{2}+\Pi \cdot \tau_{1} \operatorname{sinc}\left(\tau_{1} \Delta f\right) e^{-j \pi \tau_{1} \Delta f} \\
& +\Pi \cdot \frac{1}{\gamma+j 2 \pi \Delta f} e^{-j 2 \pi \tau_{1} \Delta f} .
\end{aligned}
$$

Coherence bandwidths are determined by solving numerically for the frequency separations, where the correlation function's magnitude drops to 0.5 or 0.9 .

\footnotetext{
${ }^{4}$ The authors would like to thank Dr. G. J. M. Janssen for providing them with measurement results for the validation of the proposed methods [9]. The measurements were conducted at the TNO Physics and Electronics Laboratory in The Hague, The Netherlands, in the period of August-December 1991.
}
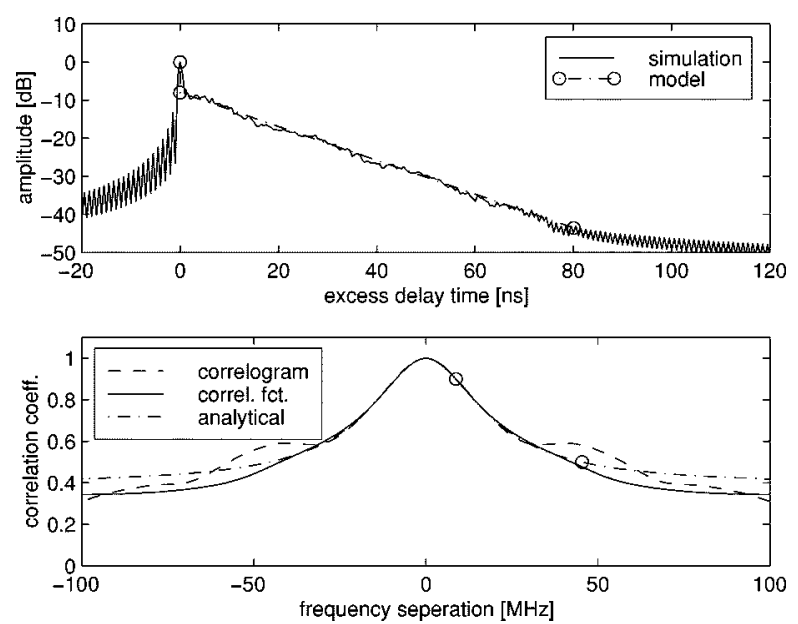

Fig. 5. Delay power spectrum (DPS) and spaced-frequency correlation function for the FD-simulation model. Upper plot: DPS and estimated power spectrum. Lower plot: Correlogram; estimated and analytical correlation functions; markers "o": Coherence bandwidths 0.5 and 0.9 .

\section{ANALYSIS OF THE FD-MODEL}

This section first presents relations between the model parameters and the main (physical) channel parameters. The rest of the section investigates the frequency-domain level crossing rate. Some novel properties are found, which are of practical interest to channel measurements and air-interface design.

\section{A. (Physical) Channel Parameters}

For the application of the channel model introduced in Section II, it is most important to relate its parameters $\left\{\rho^{2}, \Pi, \gamma, \tau_{1}\right\}$ to (physical) channel parameters. The set of channel parameters considered includes the normalized received power $P_{0}$, the Ricean $\mathrm{K}$-factor $K$, and the rms delay spread $\tau_{\text {rms }}$. A very strong indication for the importance of this particular set of parameters is seen from the analysis of the FD-level crossing rate later in this section.

The channel parameters derived from the channel model are statistical mean values, defining the behavior of the (quasi) WSSUS channel within a local area (see footnote 1). Finite bandwidth realizations or measurements within a local area have "instantaneous" channel parameters spread around those means.

Table I gives an overview of expressions relating the model parameters $\left\{\rho^{2}, \Pi, \gamma, \tau_{1}\right\}$ to the channel parameters $\left\{P_{0}, K, \tau_{\text {rms }}\right\}$ and vice versa. The derivation of these equations is outlined in Appendix B. For notational convenience we introduce $u_{1}=u+1, u_{2}=u^{2} / 2+u+1$, and $u_{3}=u^{3} / 3+u^{2}+2 u+2$, where $u=\tau_{1} \gamma$ is expressing the "shape" of the DPS (see above). An important special case is given by $u=0$, the exponentially decaying DPS, which is an appropriate description for many practical channels. Table I also lists the simplified expressions for this case.

\section{B. Level Crossing Statistics in the Frequency-Domain}

The level crossing rate (LCR) is usually defined for timedomain fading as the average number of crossings per second at which the envelope of a signal $\xi(t)$ crosses a specified level $r$ in a positive direction. Its dimension is $[1 / \mathrm{s}]$. 
TABLE I

Relation Between Model and Channel Parameters. (The Symbols are Defined in the TeXT)

\begin{tabular}{|l|l||l|l|}
\hline \multicolumn{2}{|c||}{ model $\rightarrow$ channel } & \multicolumn{2}{c|}{ channel $\rightarrow$ model } \\
\hline$u=\tau_{1} \gamma \in[0, \infty]$ & $u=0$ & $u=\tau_{1} \gamma($ must be known) & $u=0$ \\
\hline$P_{0}=\rho^{2}+\frac{\Pi}{\gamma} u_{1}$ & $P_{0}=\rho^{2}+\frac{\Pi}{\gamma}$ & $\rho^{2}=P_{0} \frac{K}{K+1}$ & $\rho^{2}=P_{0} \frac{K}{K+1}$ \\
\hline$K=\frac{\rho^{2} \gamma}{\Pi u_{1}}$ & $K=\frac{\rho^{2} \gamma}{\Pi}$ & $\gamma=\frac{1}{\tau_{r m s}} \sqrt{\frac{1}{K+1} \frac{u_{3}}{u_{1}}-\frac{1}{(K+1)^{2}} \frac{u_{2}{ }^{2}}{u_{1}{ }^{2}}}$ & $\gamma=\frac{1}{\tau_{r m s}} \frac{\sqrt{2 K+1}}{K+1}$ \\
\hline$\tau_{r m s}=\frac{1}{\gamma} \sqrt{\frac{1}{K+1} \frac{u_{3}}{u_{1}}-\frac{1}{(K+1)^{2}} \frac{u_{2}{ }^{2}}{u_{1}{ }^{2}}}$ & $\tau_{r m s}=\frac{1}{\gamma} \frac{\sqrt{2 K+1}}{K+1}$ & $\Pi=\frac{P_{0}}{K+1} \cdot \frac{\gamma}{u_{1}}$ & $\Pi=\frac{P_{0}}{K+1} \gamma$ \\
\hline
\end{tabular}

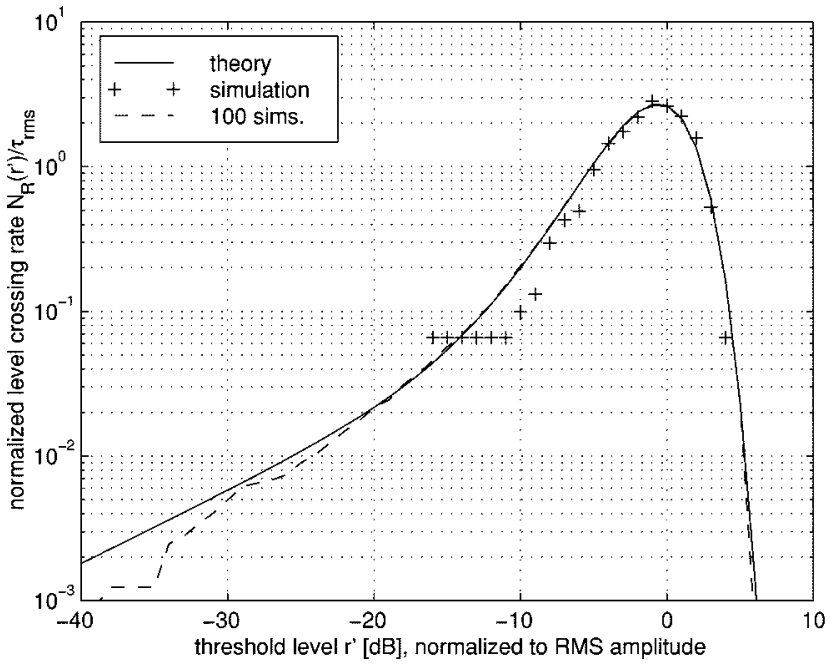

(a)

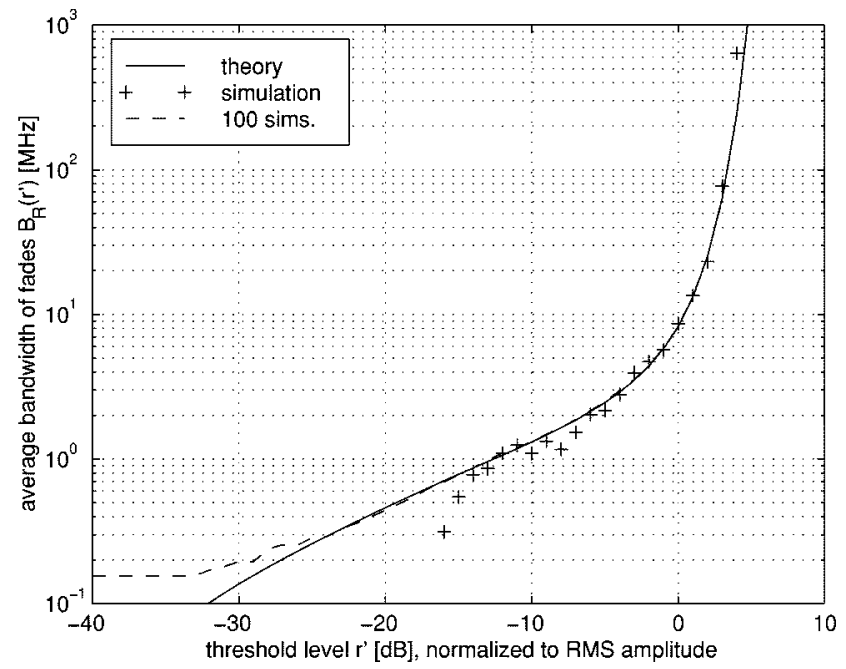

(b)

Fig. 6. (a) Normalized level crossing rate for Ricean $\mathrm{K}$-factor $K=7.5 \mathrm{~dB}$. Analytical results compared with results from one single simulation and averaged results from 100 simulations (simulated bandwidth $1.28 \mathrm{GHz} ; \tau_{\mathrm{rms}}=25.3 \mathrm{~ns}$ ). (b) Average bandwidth of fades for the same simulations.

Considering the TF in the frequency-domain, the $\mathrm{LCR}_{\mathrm{f}}$ gives the average number of crossings per Hertz bandwidth at which the amplitude $R(f)=|H(f)|$ of the TF crosses a level $r$ in a positive direction. This $\mathrm{LCR}_{\mathrm{f}}$ will be denoted by $N_{R}(r)$, its dimension being [s].

It is shown in Appendix C that-for the FD-model-the $\mathrm{LCR}_{\mathrm{f}}$ is proportional to $\tau_{\mathrm{rms}}$, denoted as

$$
N_{R}\left(r^{\prime}\right)=\tau_{\text {rms }} \cdot f\left(K, u, r^{\prime}\right)
$$

where $r^{\prime}=r / \sqrt{P_{0}}$ is the normalized threshold variable.

This result can be used for estimating $\tau_{\text {rms }}$ from the $\mathrm{LCR}_{\mathrm{f}}$, which can be obtained from noncoherent wide-band measurements (i.e., wide-band power measurements) [4]. It enables the wide-band characterization of the radio channel using a very simple measurement principle, as explained in the introduction. Section $\mathrm{V}$ discusses this method in detail.

The average bandwidth of fades (ABF), $B_{R}(r)$, is the mean value for the bandwidth over which the amplitude $R(f)$ of the TF is below a specified level $r$. Equivalently to the respective time-domain parameter (the average duration of fades [1]), the $\mathrm{ABF}$ is written as

$$
B_{R}(r)=\frac{P_{R}(r)}{N_{R}(r)}
$$

where $P_{R}(r)$ denotes the (Ricean) cdf of the signal envelope, i.e., the probability that $R(f)$ is below the level $r$.

$$
\begin{aligned}
P_{R}(r) & =\operatorname{Pr}\{R(f) \leq r\} \\
& =\frac{1}{\psi_{0}} e^{-\rho^{2} / 2 \psi_{0}} \int_{0}^{r} x e^{-x^{2} / 2 \psi_{0}} I_{0}\left(\frac{x \rho}{\psi_{0}}\right) d x .
\end{aligned}
$$

In this equation, $I_{0}(\bullet)$ designates the zeroth-order modified Bessel function of the first kind, and $\psi_{0}=(1 / 2) \Pi u_{1} / \gamma$ (see Appendix C).

Computational results of the normalized level crossing rate and the average bandwidth of fades are shown in Fig. 6(a) and (b), respectively, and are compared to computer simulations using the FD-simulation scheme. The comparison clearly demonstrates that the analytical expressions describe the statistical properties of the simulated channel appropriately.

\section{Influence of Channel and Model Parameters}

Using the analytical expressions, the influence of the channel parameters $\left\{P_{0}, K, \tau_{\mathrm{rms}}\right\}$ and the influence of the shape of the DPS (expressed by $u=\tau_{1} \gamma$ ) on the results of $\mathrm{LCR}_{\mathrm{f}}$ and ABF is studied.

One of the main results is that the $\mathrm{LCR}_{\mathrm{f}}$ is proportional to $\tau_{\text {rms }}$. For this reason it is appropriate to show $\mathrm{LCR}_{\mathrm{f}}$ and $\mathrm{ABF}$ 


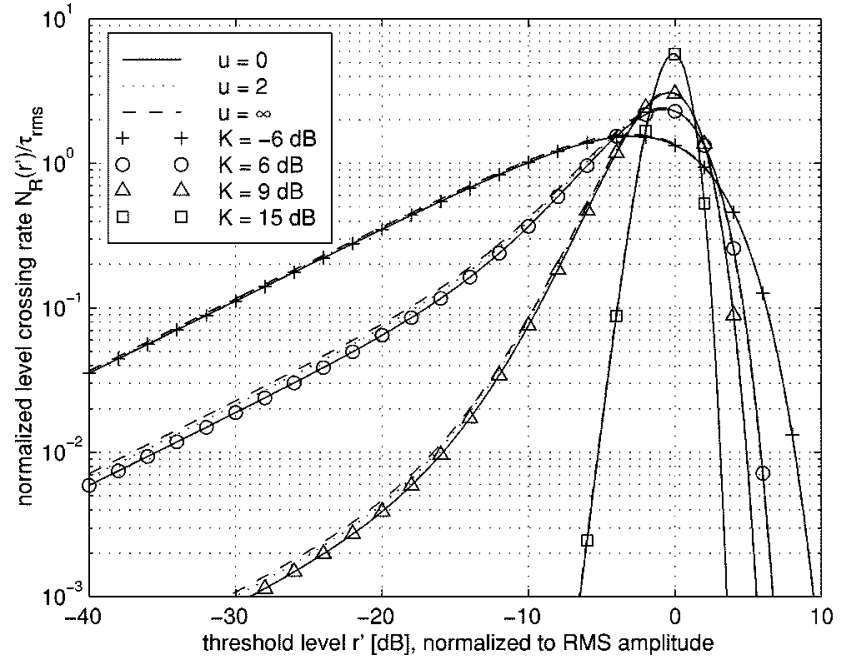

(a)

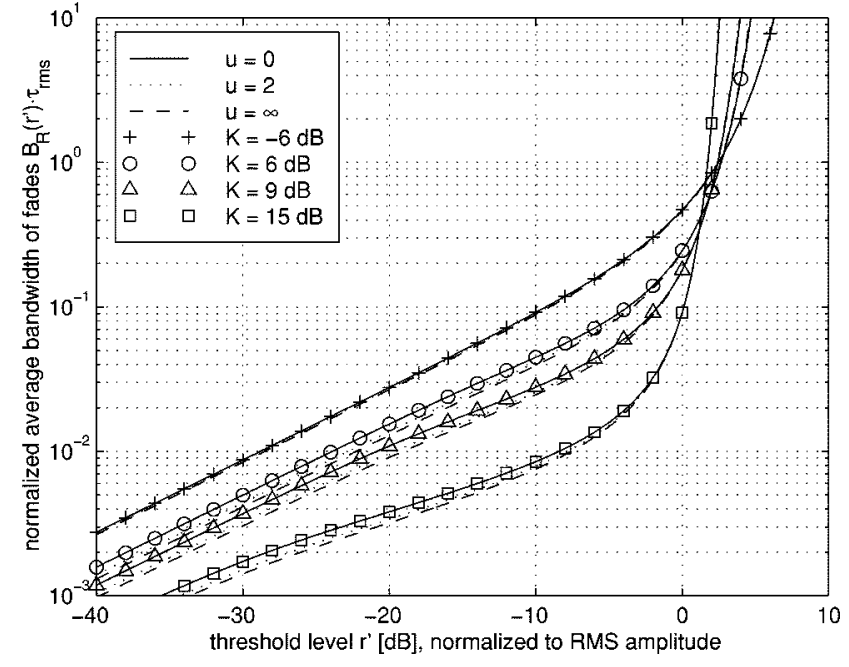

(b)

Fig. 7. (a) $\mathrm{LCR}_{\mathrm{f}}$ for various K-factors and parameters $u$. (b) $\mathrm{ABF}$ for the same parameters. Both figures are normalized to $\tau_{\text {rmas }}$ and $\sqrt{P_{0}}$. Little influence of the shape of the delay power spectrum (expressed by the parameter $u$ ) is observed.

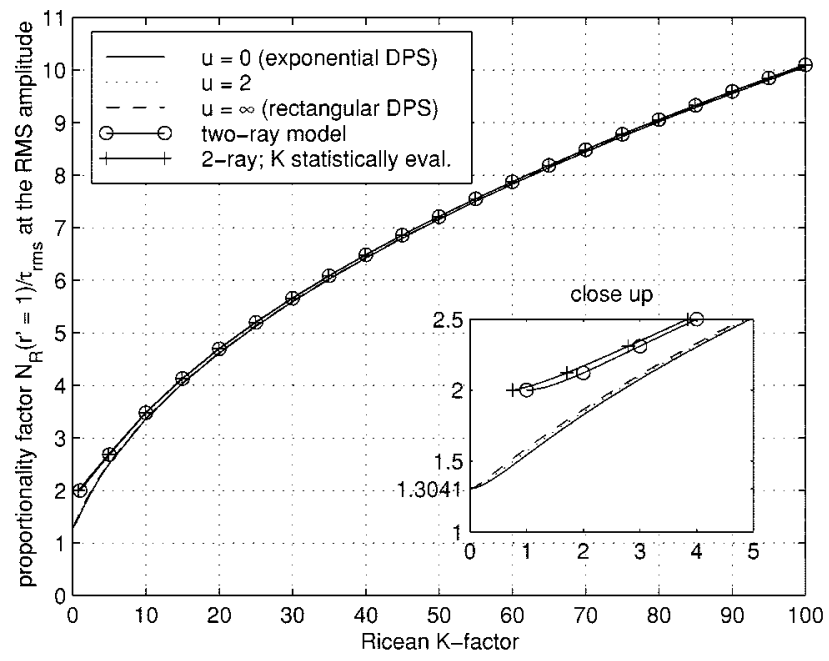

(a)

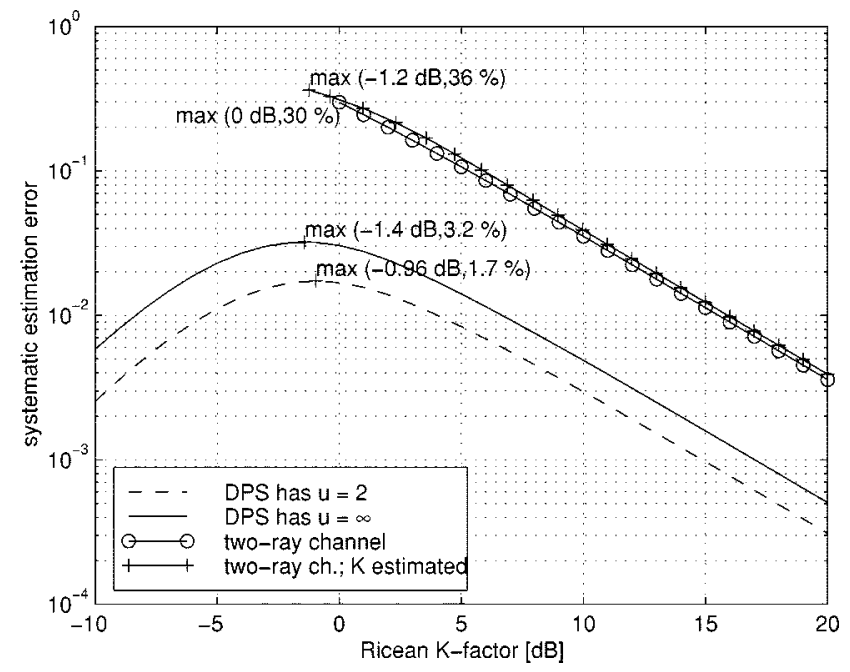

(b)

Fig. 8. (a) The factor $N_{R}\left(r^{\prime}=1\right) / \tau_{\text {rms }}$ as a function of the Ricean K-factor for various channel models. (b) Estimation error of $\tau_{\text {rms }}$, when $f(K, u=0,1)$ is used, but the channels are characterized by other models.

normalized with $\tau_{\text {rms }}$. Furthermore, the result is independent of the NRP $P_{0}$, if the threshold variable $r$ is normalized to $\sqrt{P_{0}}$. As a result, the shape of the $\mathrm{LCR}_{\mathrm{f}}$ is characteristic for particular K-factors (and parameters $u$ ) as seen from the factor $f\left(K, u, r^{\prime}\right)$ in (5). Normalized $\mathrm{LCR}_{\mathrm{f}}$ and $\mathrm{ABF}$ are depicted in Fig. 8(a) and (b), respectively, as a function of $r^{\prime}$ and for various K-factors and parameters $u$.

It is observed from these figures that even in the extreme cases $u=0$ and $u=\infty$, the $\mathrm{LCR}_{\mathrm{f}}$ and $\mathrm{ABF}$ are hardly influenced, provided $\tau_{\mathrm{rms}}$ and $K$ are kept constant. Especially the $\mathrm{LCR}_{\mathrm{f}}$ at $r^{\prime}=1\left(r=\sqrt{P_{0}}\right)$ does not show much variation, which can also be seen from Fig. 7(a) illustrating the factor $f\left(K, u, r^{\prime}=\right.$ $1)$ as a function of $K$ with parameter $u$. The dependency on $u$ disappears completely for $K=0$, i.e., for Rayleigh fading channels [16].

From the behavior of the $\mathrm{LCR}_{\mathrm{f}}$-curves, conclusions are drawn on the significance of the channel parameters $\left\{P_{0}, K, \tau_{\mathrm{rms}}\right\}$ used in this paper. Each of them has a very different impact on the $\mathrm{LCR}_{\mathrm{f}}$, thus one might expect them to have different impact on performance results as well. Clearly, the distribution of the signal-to-noise ratio is related to $P_{0}$ and $K$, which basically determines the bit error rate (BER) achievable.

Considering multicarrier transmission systems (OFDM), one would furthermore expect a dependency of the BER on the number of fades within the transmission bandwidth. From the $\mathrm{LCR}_{\mathrm{f}}$, this parameter is seen to be strictly related to $\tau_{\mathrm{rms}}$. Performance evaluations of OFDM systems have confirmed this observation [12], [13].

For (nonequalized) single-carrier modulation schemes, the relationship between $\tau_{\text {rms }}$ and the BER is even more obvious, since the delay spread determines the amount of intersymbol-interference, which itself impacts on irreducible error floors. $\mathrm{Nu}-$ merous studies are available for various modulation and detection schemes, reporting on qualitative and quantitative relations between $\tau_{\text {rms }}$ and the BER. Although no general result is known, $\tau_{\text {rms }}$ is probably the most important single parameter 
for characterizing the time-dispersion or frequency-selectivity of the wide-band radio channel.

\section{Level Crossing Rate for a Two-Ray Model}

To confirm the significance of the proportionality factor relating the $\mathrm{LCR}_{\mathrm{f}}$ to $\tau_{\mathrm{rms}}$, a deterministic two-ray model is investigated in this section. The IR of such a channel is defined as

$$
h(\tau)=\beta_{0} e^{j \theta_{0}} \delta(\tau)+\beta_{1} e^{j \theta_{1}} \delta\left(\tau-\tau_{\delta}\right)
$$

where

$$
\begin{array}{ll}
\beta_{0} \geq \beta_{1} & \text { ray amplitudes; } \\
\theta_{0,1} & \text { ray phases; } \\
\tau_{\delta}>0 & \text { relative delay among the two paths. }
\end{array}
$$

Applying the FT leads to the amplitude TF

$$
R(f)=|H(f)|=\sqrt{\beta_{0}^{2}+\beta_{1}^{2}+2 \beta_{0} \beta_{1} \cos 2 \pi \tau_{\delta} f} .
$$

From (9), the $\mathrm{LCR}_{\mathrm{f}}$ is seen to be constant:

$$
N_{R}(r)= \begin{cases}\tau_{\delta} & \beta_{0}-\beta_{1} \leq r \leq \beta_{0}+\beta_{1} \\ 0 & \text { otherwise }\end{cases}
$$

$\tau_{\text {rms }}$ must be calculated for this model to obtain the normalized $\mathrm{LCR}_{\mathrm{f}}$, which is the proportionality factor required. Analyzing the IR yields

$$
\tau_{\text {rms }}=\tau_{\delta} \frac{\beta_{0} \beta_{1}}{\beta_{0}^{2}+\beta_{1}^{2}}=\tau_{\delta} \frac{\sqrt{\kappa}}{\kappa+1}
$$

where $\kappa=\beta_{1}^{2} / \beta_{0}^{2}$ is the power ratio of the two rays. Taking $\kappa$ as K-parameter, (10) and (11) can be used to derive the proportionality factor as a function of $K$ [see Fig. 8(a), "o-o"].

In fact, the Ricean distribution is not describing the amplitude distribution of (9), thus comparing $\kappa$ to the Ricean K-factor in (5) might be inappropriate. One method of deriving $K$ from a set of amplitude values $R$ is to calculate $E\{R)$ and $E\left\{R^{2}\right\}$. The ratio $E\{R\} / \sqrt{E\left\{R^{2}\right\}}$ can be related to $K$ as elaborated in [17]. Using this definition of $K$, the proportionality factor is found as indicated by " $+\ldots+$ " in Fig. 8(a).

The similarity of all the results shown in this graph confirms the significance of the relationship found between $\tau_{\text {rms }}$ and the $\mathrm{LCR}_{\mathrm{f}}$. This suggests that the proposed measurement method can be applied quite generally, i.e., even if the investigated propagation channel does not fully match to the model defined in Fig. 1. Recent work has shown that, for Rayleigh fading channels, the dependency on the model disappears completely [16].

In Fig. 8(b), the error of $\tau_{\text {rms }}$ is depicted, resulting from strictly using $f\left(K, u=0, r^{\prime}=1\right)$ when estimating $\tau_{\text {rms }}$ for channels described by other models (FD-model for $u=\{2, \infty\}$ and two-ray model). In case of the FD-model with a rectangular DPS $(u=\infty)$, the maximum error is $3.2 \%$ at $K=-1.4 \mathrm{~dB}$. If a two-ray channel is evaluated with this method, the maximum error is $36 \%$, when both rays have equal powers and it drops below $10 \%$ when $\kappa$ is higher than $6 \mathrm{~dB}$.

\section{Using the Level Crossing Rate for Channel MEASUREMENTS}

\section{A. Description of the Method}

Exploiting the proportionality of the $\mathrm{LCR}_{\mathrm{f}}$ and $\tau_{\mathrm{rms}}$, the rms delay spread can be estimated from noncoherent wide-band channel measurements, where the received signal strength is measured as a function of the frequency. The analysis of such measurements requires several steps.

First, the Ricean K-factor $\hat{K}$ has to be derived from the measured amplitude TF $R(f)=|H(f)|$. This can be achieved, e.g., from the mean and rms values of the amplitudes $E\{R\}$ and $\sqrt{E\left\{R^{2}\right\}}$ as described in [17] (see also Section IV-D). Then an estimate of the RDS $\left(\hat{\tau}_{\text {rms }}\right)$ is obtained by taking the $\mathrm{LCR}_{\mathrm{f}}$ at $r=\sqrt{P_{0}}=\sqrt{E\left\{R^{2}\right\}}, \hat{N}_{R}\left(r^{\prime}=1\right)$, and evaluating (5) for $f\left(K=\hat{K}, u=0, r^{\prime}=1\right)$ as given in Fig. 8(a). For measurements, the following approximation of $f\left(K, u, r^{\prime}\right)$ at $u=0$ and $r^{\prime}=1$ can be used, which results in errors below $1 \%$ for all values of $K$, compared to the exact $f(K, 0,1)$

$$
\tilde{f}(K, 0,1)= \begin{cases}\frac{1}{4} K^{3 / 2}+1.3041, & K \leq 1 \\ \sqrt{K} \frac{K+1}{K+0.31}, & K>1\end{cases}
$$

The accuracy obtained depends heavily on the observation bandwidth, since the measurement method is based on a statistical model. By increasing the bandwidth, the number of detected level crossings is increased, and thus the accuracy of the estimated RDS is enhanced. A valuable advantage of the proposed method is found in the fact that-because of ergodicity - the observation bandwidth can be increased not only by increasing the bandwidth of the measurement, but also by combining data from several measurements that are performed within a local area. This local area must be sufficiently small (maximum size $\cong 5 \ldots 40 \lambda$ ) so that we can assume the channel parameters (and thus the statistical properties of the channel) to be constant. In other words, the shadowing must be constant. Data collected for such a cluster of measurements is analyzed as follows. The NRP and the K-factor are determined by simply combining all measured amplitudes and calculating $E\{R\}$ and $\sqrt{E\left\{R^{2}\right\}}$ for the resulting data set. Level crossing rates must be calculated for each measurement separately. Consecutively, they are averaged to obtain $\hat{N}_{R}(r)$ for the combined data set.

The proposed method was evaluated using channel measurements performed with a network analyzer, and using time-domain channel simulations.

\section{B. Measurement Results}

Coherent measurements, as obtained with a network analyzer, allow the calculation of the channel's IR from which a reference-value of $\tau_{\text {rms }}$ can be calculated. Clusters of six measurements were investigated within the local areas (of diameter $=2 \lambda$ ), where each TF had $1-\mathrm{GHz}$ bandwidth around a center frequency of $11.5 \mathrm{GHz}$ [9] (see footnote 3).

Fig. 9(a) shows the empirical $\mathrm{LCR}_{\mathrm{f}}$ of one measured TF as a function of $r^{\prime}$ and the analytical curve for the estimated parameters $\hat{K}$ and $\hat{\tau}_{\text {rms }}\left(\tau_{1}=0\right)$. The good agreement demonstrates the 


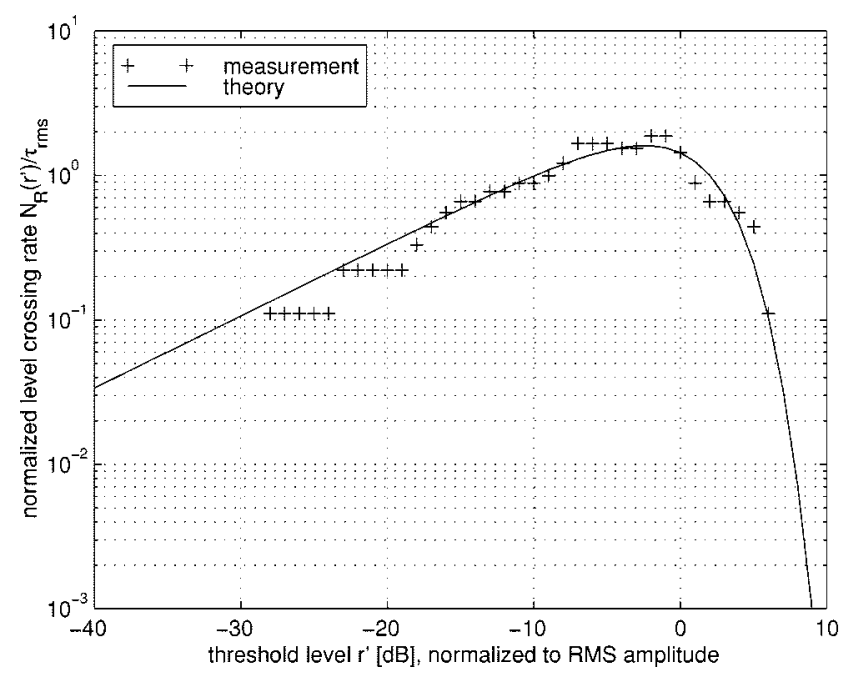

(a)

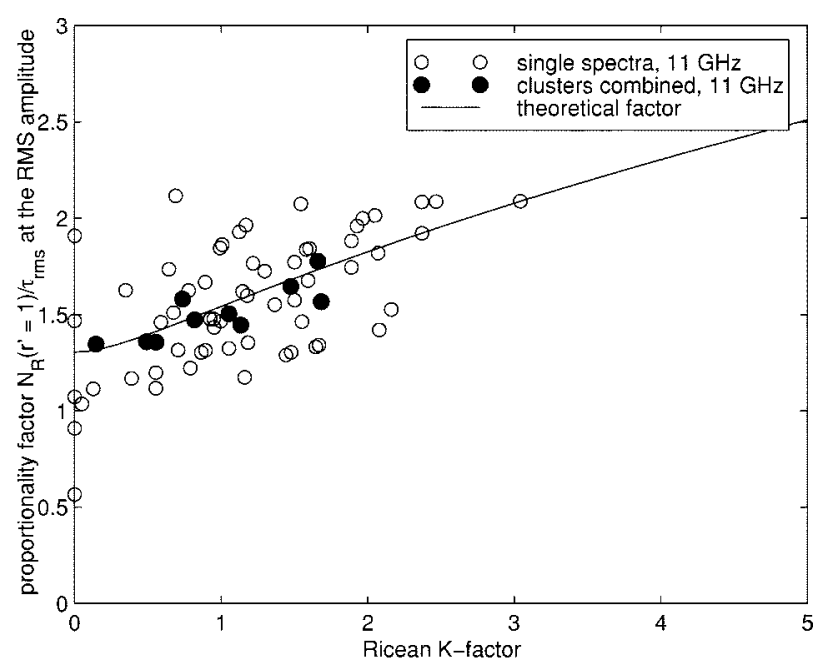

(b)

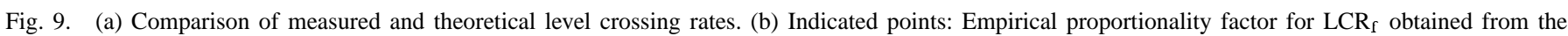
amplitude TF and $\tau_{\text {rms }}$ obtained from the IR. Curve ("- "): Theoretical factor $f\left(K, u=0, r^{\prime}=1\right)$. The mismatch indicates the estimation error.

suitability of the FD-model for characterizing the frequency-selective channel.

To assess the accuracy of the estimated RDS, the proportionality factor $\hat{N}_{R}\left(r^{\prime}=1\right) / \tau_{\text {rms }}$ is depicted in Fig. 9(b), where $\hat{N}_{R}\left(r^{\prime}=1\right)$ and $\hat{K}$ were derived from the amplitude TF $R(f)=$ $|H(f)|$ and (the reference) $\tau_{\text {rms }}$ was calculated from the channel IR [18], [10]. The RDS was estimated for every single measured $\mathrm{TF}$ and also for the combined data sets of each cluster of measurements. The theoretical factor $N_{R}\left(r^{\prime}=1\right) / \tau_{\text {rms }}=$ $f(K, 0,1)$ is shown in the same figure for comparison. The distance between this curve and the data points indicates the estimation error. It is observed from this figure that the estimation error is decreased significantly by investigating the combined data of the measurement clusters.

\section{Time-Domain Simulations}

Channel simulations obtained from a time-domain (TD) simulation scheme were analyzed for evaluating the estimation error as a function of the simulated (=observed) bandwidth. This approach has been taken because the RDS can be calculated exactly from the channel's IR-the output of the TD simulator-which provides us with the reference value $\tau_{\text {rms }}$. The TD simulation model assumes a Poisson process of ray-arrivals, an exponentially decaying average power delay profile, Rayleigh distributed ray amplitudes, and uniformly distributed ray phases ([10]; for 1 cluster). Applying the Fourier transform to the generated IR, amplitude TFs were obtained. Consecutively, the rms delay spreads were estimated using the proposed procedure, yielding $\hat{\tau}_{\text {rms }}$. The estimation error is defined as

$$
\varepsilon=\frac{\hat{\tau}_{\mathrm{rms}}-\tau_{\mathrm{rms}}}{\tau_{\mathrm{rms}}} .
$$

Performing sets of 100 simulations for different values of $K$ and different observation bandwidths, the mean and standard deviation of the error $\varepsilon$ was investigated. Results are depicted in Fig. 10(a), as a function of the normalized observation bandwidth. It was observed that the Gaussian distribution appropriately describes the pdf of $\varepsilon, p_{\varepsilon}(x)$.

Using the estimation method described above (standard method; est. 1), a small systematic estimation error of approximately $-3 \%$ and decreasing standard deviation with increased bandwidth can be seen from the results (bold lines). An enhanced estimation method (est. 2) determines $\tau_{\text {rms }}$ from the level crossing rates at several threshold values $r$, thereby reducing the systematic error and improving the accuracy [Fig. 10(a), thin lines]. An optimization of these methods is subject for further research.

In Fig. 10(b), the standard deviation of the error is shown as a function of the number of level crossings. This diagram clearly reveals that the number of observed level crossings determines the estimation accuracy.

\section{Discussion of the Measurement Method}

It is seen that the RDS can be estimated with reasonable accuracy when the observation bandwidth is larger than $10 / \tau_{\text {rms }}$, or when more than ten level crossings are present. While the required bandwidth might be higher than the bandwidth needed for other measurement techniques, it can be increased easily by combining multiple measurements performed within a small local area. Another advantage lies in the simplicity of the hardware used. It makes the method particularly interesting at extremely high frequencies (millimeter wave band), where, e.g., network analyzers become very cumbersome due to the phase reference required, which is very difficult to provide over large distances. Suitable network analyzers are also extremely expensive. A clear advantage compared to other channel sounding techniques is that no specific equipment must be designed. A continuous wave frequency generator and a power meter or spectrum analyzer may be used to collect measurement data.

Preliminary measurement campaigns performed at Delft University of Technology [19]-[22] have shown the practical suitability of the described methods. But they have also led to the 


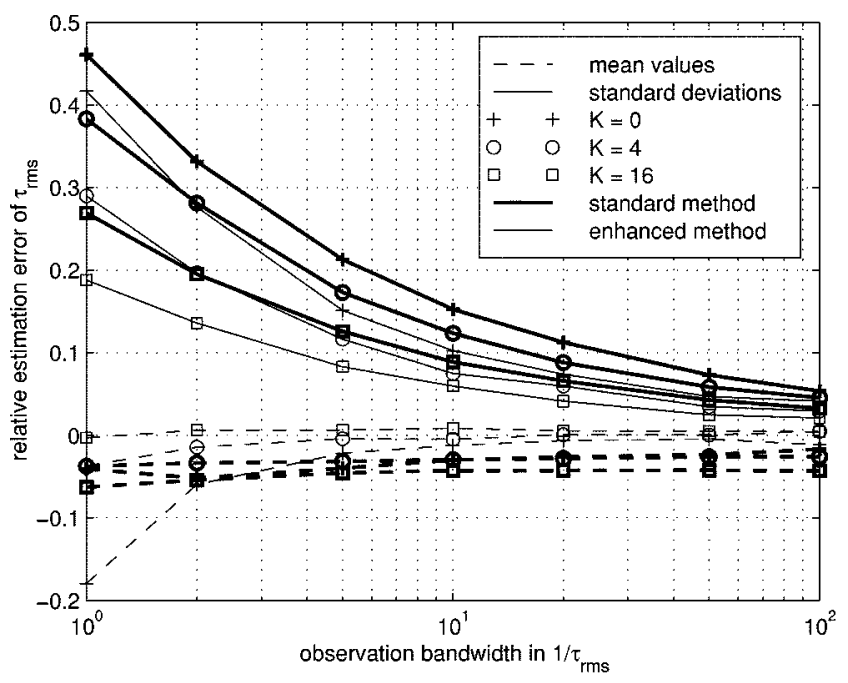

(a)

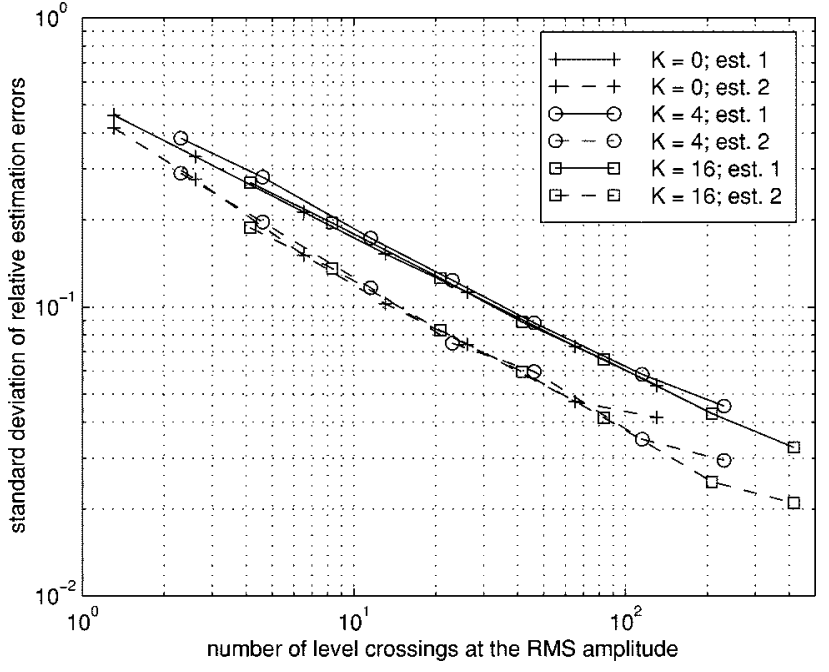

(b)

Fig. 10. Relative estimation error of $\tau_{\text {rms }}$, derived from sets of hundred TD simulations. (a) Mean and standard deviation of the estimation error $\varepsilon$ as a function of the normalized bandwidth. (b) Standard deviation of the estimation $\varepsilon$ error as a function of the number of level crossings.

discovery of another problem. Even small measurement noise may increase the number of level crossings detected. Improved measurement methods have been proposed in these references to reduce this problem. The theoretical analysis [23] shows that the frequency-domain sampling interval of the measurements should be selected as large as possible to minimize the noise's impact. This issue is still under investigation (see also [24]).

\section{CONCLUSION AND RECOMMENDATIONS}

An elaborate study of the frequency-selective (= time-dispersive) radio channel is presented. The study is based on a stochastic channel model, called the frequency-domain (FD) model. The emphasis lies on time-invariant channels with Rayleigh or Ricean amplitude distributions. Just as the time-variability of a narrowband time-variant channel is characterized by the Doppler spectrum (and the spaced-time correlation function), the frequency-selectivity is characterized by the delay power spectrum (and the spaced-frequency correlation function).

A simulation scheme is shown, which is dual to the well-known simulation schemes for narrowband time-variant channels. Essentially, shaping the spectrum of a complex Gaussian process generates the wide-band channel's transfer function (TF). This is of particular interest when the simulation result is needed in the FD, which-for instance-may be the case for the investigation of OFDM systems.

The model allows for elaborate mathematical analysis. Simple expressions relate model and relevant channel parameters. They can be employed to fit the FD-model to any set of (measured) channel parameters.

The level crossing rate $\left(\mathrm{LCR}_{\mathrm{f}}\right)$ and average bandwidth of fades of the amplitude transfer function (TF) $R(f)=|H(f)|$ are defined and expressed analytically. It was observed that the rms delay spread (RDS) is proportional to the $\mathrm{LCR}_{\mathrm{f}}$. Applying this property, the RDS can be estimated from noncoherent wide-band measurements (received power vs. frequency), leading to a simplified channel measurement technique. The accuracy of this technique depends on the bandwidth observed. It can be increased by combining multiple measurements performed within a small local area.

The influence of model and channel parameters on the level crossing statistics was investigated. Average power, Ricean $\mathrm{K}$-factor, and RDS each have different impacts on the $\mathrm{LCR}_{\mathrm{f}}$. The actual channel model changes the $\mathrm{LCR}_{\mathrm{f}}$ insignificantly. From these observations it is concluded that the RDS is an important parameter for characterizing the time-dispersitivity of the radio channel.

\section{APPENDIX A}

This appendix explains the derivation of the variance $\sigma_{W}^{2}$ of the noise source in the FD-simulation scheme shown in Fig. 2. The model of the DPS, the spectrum of the TF in the excess delay time domain [see (3)], must match with the PSD of the generated sequence $H\left(n f_{s}\right) .\left(f_{s}[\mathrm{~Hz}]\right.$ is the sampling interval of the TF.)

The noise source produces independent, real-valued samples with variance $\sigma_{W}^{2}$. The sequence $W\left(n f_{s}\right)$ thus has a (periodic) spectrum with constant PSD

$$
S_{W}(\tau)=\sigma_{W}^{2} f_{s}
$$

As mentioned in Section III-B, the noise shaping filter $g(f)$ is designed to be proportional to the DPS, with TF $|G(\tau)|=1$ during the constant level part, i.e.,

$$
|G(\tau)|= \begin{cases}1, & |\tau| \leq \tau_{1} \\ e^{-\gamma\left(|\tau|-\tau_{1}\right)}, & |\tau|>\tau_{1}\end{cases}
$$

leading to the PSD of $\operatorname{Re}\left\{H^{\prime}(n f s)\right\}$ written as

$$
S_{r} H^{\prime}(\tau)=\sigma_{W}^{2} f_{s}|G(\tau)|
$$

The next step in the simulation scheme is the addition of the Hilbert transformed (HT) sequence, which increases the PSD for $\tau>0$ by a factor of four. (The HT cancels the negative- $\tau$ part of the Fourier spectrum, while doubling the positive- $\tau$ part, 
resulting in four-fold power for $\tau>0$.) This yields the PSD to be compared with the model (the DPS) as

$$
S_{H^{\prime}}(\tau)=4 \sigma_{W}^{2} f_{s}|G(\tau)| \equiv \phi_{h}(\tau), \quad \text { for } \tau>0
$$

which gives $\sigma_{W}^{2}=\Pi /\left(4 f_{s}\right)$.

\section{APPENDIX B}

From the continuous DPS $\phi_{h}(\tau)$ defined by (3), analytical expressions can be derived for the expected values of normalized received power $P_{0}$, Ricean K-factor $K$, and $\operatorname{RDS} \tau_{\text {rms }} . P_{0}$ relates to the DPS as

$$
E\left\{P_{0}\right\}=\int_{0}^{\infty} \phi_{h}(\tau) d \tau=\rho^{2}+\Pi \cdot\left[\tau_{1}+\frac{1}{\gamma}\right] .
$$

The K-factor is used to characterize the amplitude distribution of Ricean channels, relating the power of the direct path to the power of the scattered paths.

$$
E\{K\}=\frac{\rho^{2}}{E\left\{P_{0}\right\}-\rho^{2}}=\frac{\rho^{2}}{\Pi \cdot\left(\tau_{1}+1 / \gamma\right)} .
$$

$\tau_{\text {rms }}$ is the single most important parameter characterizing the frequency-selectivity. It can be interpreted as the centralized second moment of the normalized DPS

$$
E\left\{\tau_{\mathrm{rms}}\right\}=\sqrt{E\left\{\overline{\tau^{2}}\right\}-(E\{\bar{\tau}\})^{2}}
$$

where

$$
\begin{aligned}
E\{\bar{\tau}\} & =\int_{0}^{\infty} \tau \frac{\phi_{h}(\tau)}{E\left\{P_{0}\right\}} d \tau=\Pi \cdot\left[\frac{\tau_{1}^{2}}{2}+\frac{\tau_{1}}{\gamma}+\frac{1}{\gamma^{2}}\right] \\
E\left\{\overline{\tau^{2}}\right\} & =\int_{0}^{\infty} \tau^{2} \frac{\phi_{h}(\tau)}{E\left\{P_{0}\right\}} d \tau=\Pi \cdot\left[\frac{\tau_{1}^{3}}{3}+\frac{\tau_{1}^{2}}{\gamma}+\frac{2 \tau_{1}}{\gamma^{2}}+\frac{2}{\gamma^{3}}\right] .
\end{aligned}
$$

For notational convenience, the expectation operators are skipped in the main text. $\left\{P_{0}, K, \tau_{\text {rms }}\right\}$ always denote expected channel parameters, unless otherwise specified.

\section{APPENDIX C}

An analytical expression for the LCR of Ricean processes with cross-correlated real- and imaginary parts of the underlying complex Gaussian process $H^{\prime}(f)$ is given as (compare [25])

$$
\begin{aligned}
N_{R}(r)= & \frac{r \sqrt{2 \beta}}{\pi^{3 / 2} \psi_{0}} e^{-\left(r^{2}+\rho^{2}\right) /\left(2 \psi_{0}\right)} \int_{0}^{\pi / 2} \cosh \left(\frac{r \rho}{\psi_{0}} \cos \theta\right) \\
& \times\left\{e^{-(\alpha \rho \sin \theta)^{2}}+\sqrt{\pi} \alpha \rho \sin (\theta) \operatorname{erf}(\alpha \rho \sin \theta)\right\} d \theta
\end{aligned}
$$

where $\psi_{0}=(1 / 2) \phi_{H^{\prime}}(0)=(1 / 2) \Pi\left[\tau_{1}+1 / \gamma\right]$ is the variance of the real or imaginary component of $H^{\prime}(f)$ (i.e., $1 / 2$ the power of the scattered rays), and $\alpha=-\dot{\phi}_{0} /\left(\psi_{0} \sqrt{2 \beta}\right)$ and $\beta=-\ddot{\psi}_{0}-\dot{\phi}_{0}^{2} / \psi_{0}$ account for the second order statistics of $H^{\prime}(f) . H^{\prime}(f)$ is defined by the DPS [(3)] after subtraction of the LOS-component $\rho^{2} \delta(\tau)$, or equivalently by its autocorrelation function $(\mathrm{ACF})$

$$
\begin{aligned}
& \phi_{H^{\prime}}(\Delta f)=F\left\{\phi_{h^{\prime}}(\tau)\right\} \\
&=\Pi\left[\tau_{1} \operatorname{sinc}\left(\tau_{1} \Delta f\right) e^{-j \pi \tau_{1} \Delta f}\right. \\
&\left.\quad+\frac{1}{\gamma+j 2 \pi \Delta f} e^{-j 2 \pi \tau_{1} \Delta f}\right] .
\end{aligned}
$$

The curvature of the ACF $\phi_{r^{\prime}}(\Delta f)$ and the gradient of the cross-correlation function (CCF) $\phi_{r H^{\prime} i^{H^{\prime}}}(\Delta f)$ have to be evaluated at $\Delta f=0$ to calculate $\alpha$ and $\beta$, where $\phi_{H^{\prime}}(\Delta f)=$ $2\left[\phi_{r^{H^{\prime}}}(\Delta f)+j \phi_{r^{H^{\prime}} i^{H^{\prime}}}(\Delta f)\right]$. This yields

$$
\begin{aligned}
& \ddot{\psi}_{0}=\left.\frac{d^{2}}{d \Delta f^{2}} \phi_{r^{H^{\prime}}}(\Delta f)\right|_{\Delta f=0}=-2 \pi^{2} \frac{\Pi}{\gamma^{3}} u_{3} \\
& \dot{\phi}_{0}=\left.\frac{d}{d \Delta f} \phi_{r^{H^{\prime}} i^{H^{\prime}}}(\Delta f)\right|_{\Delta f=0}=-\pi \frac{\Pi}{\gamma^{2}} u_{2} .
\end{aligned}
$$

Next, it will be shown that the $\mathrm{LCR}_{\mathrm{f}}$ for the FD-model can be expressed in the form

$$
N_{R}\left(r^{\prime}\right)=\tau_{\mathrm{rms}} \cdot f\left(K, u, r^{\prime}\right) .
$$

With (25) we get

and

$$
\alpha=\sqrt{\frac{\gamma}{\Pi}} \frac{u_{2}}{\sqrt{u_{3} u_{1}^{2}-u_{2}^{2} u_{1}}}
$$

$$
\beta=2 \pi^{2} \frac{\Pi}{\gamma^{3}}\left[u_{3}-\frac{u_{2}^{2}}{u_{1}}\right] .
$$

The threshold level $r$ must be considered relatively to the square root of the NRP, i.e.,

$$
r^{\prime}=r / \sqrt{P_{0}}
$$

Substituting (27), (28), and $\psi_{0}=(1 / 2) \Pi\left(u_{1} / \gamma\right)$ into (23) and using the expression for $\tau_{\mathrm{rms}}(u)$ from Table I yields

$$
\begin{aligned}
N_{R}\left(r^{\prime}\right)= & \tau_{\mathrm{rms}} \cdot a \cdot e^{-b} \int_{0}^{\pi / 2} \cosh (c \cdot \cos \theta) \\
& \times\left\{e^{-(d \cdot \sin \theta)^{2}}+\sqrt{\pi} d \cdot \sin \theta \cdot \operatorname{erf}(d \cdot \sin \theta)\right\} d \theta
\end{aligned}
$$

where

$$
\begin{aligned}
& a=\frac{4}{\sqrt{\pi}} r^{\prime}(K+1)^{3 / 2} \sqrt{\frac{u_{3} u_{1}-u_{2}^{2}}{u_{3} u_{1}(K+1)-u_{2}^{2}}} \\
& b=r^{\prime 2}(K+1)+K \\
& c=2 r^{\prime} \sqrt{K(K+1)} \\
& d=\sqrt{K} \frac{u_{2}}{\sqrt{u_{1} u_{3}-u_{2}^{2}}} .
\end{aligned}
$$

It is observed from (30) that $\{a, b, c, d\}$ are expressed as functions of $\left\{K, u, r^{\prime}\right\}$, i.e., they are independent of $\tau_{\text {rms }}$, which proves (26).

\section{ACKNOWLEDGMENT}

The authors would like to thank the anonymous reviewers. We are grateful to L. P. Ligthart, G. J. M. Janssen, H. Nikookar, A.-J. v.d. Veen, A. Bohdanowicz, and D. Matic from TU-Delft, and L. M. Correia from Instituto Superior Técnico (IST), Lisbon, for their comments and fruitful discussions. 


\section{REFERENCES}

[1] W. C. Jakes, Jr., Microwave Mobile Communications. New York: Wiley-Interscience, 1974.

[2] R. van Nee and R. Prasad, OFDM for Wireless Multimedia Communications. Boston, MA: Artech House, 2000.

[3] J. G. Proakis, Digital Communications, 3rd ed. New York: McGrawHill, 1995.

[4] K. Witrisal, Y.-H. Kim, and R. Prasad, "RMS delay spread estimation technique using noncoherent channel measurements," IEE Electron. Lett., vol. 34, no. 20, pp. 1918-1919, Oct. 1998.

[5] B. P. Donaldson, M. Fattouche, and R. W. Donaldson, "Characterization of in-building UHF wireless radio communication channels using spectral energy measurements," IEEE Trans. Antennas Propagat., vol. 44, pp. 80-86, Jan. 1996.

[6] P. A. Bello, "Characterization of randomly time-variant linear channels," IEEE Trans. Commun. Syst., vol. CS-11, pp. 360-393, Dec. 1963.

[7] R. Steele, Mobile Radio Communications. New York: Wiley, 1992.

[8] P. F. M. Smulders, "Broadband wireless LANs: A feasibility study," Ph.D. dissertation, Eindhoven Univ. Technol., Eindhoven, The Netherlands, 1995.

[9] G. J. M. Janssen, P. A. Stigter, and R. Prasad, "Wideband indoor channel measurements and BER analysis of frequency selective multipath channels at 2.4, 4.75 and $11.5 \mathrm{GHz}$," IEEE Trans. Commun., vol. 44, pp. 1272-1288, Oct. 1996.

[10] A. A. M. Saleh and R. A. Valenzuela, "A statistical model for indoor multipath propagation," IEEE J. Select. Areas Commun., vol. 5, pp. 128-137, Feb. 1987.

[11] O. Edfors, M. Sandell, J. J. van de Beek, D. Landström, and F. Sjöberg. An introduction to orthogonal frequency-division multiplexing. Division of Signal Processing, Luleå Univ. Technol.. [Online]. Available: http://www.sm.luth.se/csee/sp/publications.html

[12] K. Witrisal, Y.-H. Kim, and R. Prasad, "Frequency-domain simulation and analysis of the frequency-selective radio channel for the performance analysis of OFDM," in Proc. 3. OFDM Fachgespräch, Braunschweig, Germany, Sept. 1998.

[13] — , "A novel approach for performance evaluation of OFDM with error correction coding and interleaving," in Proc. VTC'99-Fall (IEEE Veh. Technol. Conf.), Amsterdam, The Netherlands, Sept. 1999, pp. 294-299.

[14] A. V. Oppenheim and R. W. Schafer, Discrete-Time Signal Processing, 2nd ed. Englewood Cliffs, NJ: Prentice-Hall, 1999.

[15] W. Zhang, "Simulation and modeling of multipath mobile channels," in Proc. VTC'94 (IEEE Veh. Technol. Conf.), Stockholm, Sweden, 1994, pp. $160-164$.

[16] K. Witrisal, "On estimating the RMS delay spread from the frequencydomain level crossing rate," IEEE Commun. Lett., vol. 5, pp. 287-289, July 2001, submitted for publication.

[17] F. van der Wijk, A. Kegel, and R. Prasad, "Assessment of a pico-cellular system using propagation measurements at $1.9 \mathrm{GHz}$ for indoor wireless communications," IEEE Trans. Veh. Technol., vol. 44, pp. 155-162, Feb. 1995.

[18] S. J. Howard and K. Pahlavan, "Autoregressive modeling of wide-band indoor radio propagation," IEEE Trans. Commun., vol. 40, pp. 1540-1552, Sept. 1992.

[19] A. Bohdanowicz, G. J. M. Janssen, and S. Pietrzyk, "Wideband indoor and outdoor multipath channel measurements at $17 \mathrm{GHz}$," in Proc. VTC'99-Fall (IEEE Veh. Technol. Conf.), Amsterdam, The Netherlands, Sept. 1999, pp. 1998-2003.

[20] A. Bohdanowicz. (2000, Jan.) Wideband indoor and outdoor radio channel measurements at $17 \mathrm{GHz}$. Delft Univ. of Technol. [Online]. Available: http://www.ubicom.tudelft.nl/docs/

[21] R. El Hattachi, J. M. M. de Nijs, K. Witrisal, and R. Prasad, "Characterization and simulation of the $18 \mathrm{GHz}$ radio channel," in Proc. IEEE Benelux 6th Symp. Veh. Technol. Commun., Brussels, Belgium, Oct. 1998.

[22] J. Purwaha, A. Mank, D. Matic, K. Witrisal, and R. Prasad, "Wide-band channel measurements at $60 \mathrm{GHz}$ in indoor environments," in Proc IEEE Benelux 6th Symp. Veh. Technol. Commun., Brussels, Belgium, Oct. 1998.

[23] K. Witrisal and A. Bohdanowicz, "Influence of noise on a novel RMS delay spread estimation method," in Proc. PIMRC 2000 (IEEE 11th Int. Symp. Personal Indoor Mobile Radio Commun.), London, U.K., Sept. 2000, pp. 560-566.
[24] K. Witrisal, G. Landman, and A. Bohdanowicz, "Practical application of a novel method of estimating the RMS delay spread from power measurements," in Proc. EPMCC 2001 (4th Euro. Mobile Commun. Conf.), Vienna, Austria, Feb. 2001.

[25] M. Pätzold, U. Killat, F. Laue, and Y. Li, "On the statistical properties of deterministic simulation models for mobile fading channels," IEEE Trans. Veh. Technol., vol. 47, pp. 254-269, Feb. 1998.

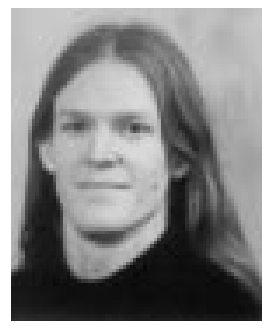

Klaus Witrisal (S'98) was born in Graz, Austria, in 1972. He received the Dipl.-Ing. in electrical engineering from Graz University of Technology in 1997.

Since 1997, he has been with Delft University of Technology, The Netherlands. For three years, he was involved in a cooperative research project on OFDM transmission techniques for wireless broadband communications in mm-wave bands, which was conducted jointly with Korea Telecom, Seoul. Currently, he is pursuing the Ph.D. degree. His research interests include the characterization of wide-band radio channels and signal processing for OFDM air-interfaces.

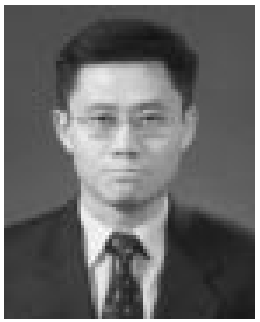

Yong-Ho Kim received the B.S. degree in electrical engineering from Han Yang University, Seoul, Korea, in 1986, the M.S. degree in electrical engineering from Korea Advanced Institute of Science and Technology (KAIST), in 1988, and the Ph.D. degree in information and communication engineering from KAIST, in 1996

From 1996 to 1998 , he was with Wireless Communications Research Lab, Korea Telecom, where he led the design and implementation of CDMA MODEM and IMT-2000 test-bed. Since 1999, he has been with Access Network Lab, Korea Telecom, where he is currently a Senior Member of Technical Staff. Since 1988, he has been with the Research and Development Group, Korea Telecom, Seoul. He is currently working on the radio access network systems of the IMT-2000, the third-generation mobile communication system as a system engineer. His main areas are the physical layer and radio access systems of the third-generation wireless communications. His research interests are wireless communication systems, CDMA MODEM, OFDM transmission technology, and signal processing.

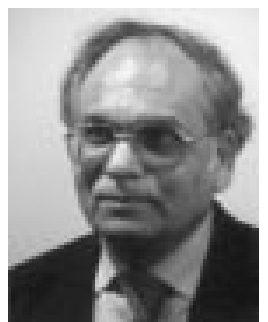

Ramjee Prasad (SM'90) was born in Babhnaur (Gaya), Bihar, India, on July 1, 1946. He is now a Dutch Citizen. He received the B.Sc. (eng) degree from Bihar Institute of Technology, Sindri, India, and the M.Sc. (eng) and Ph.D. degrees from Birla Institute of Technology (BIT), Ranchi, India, in 1968, 1970, and 1979, respectively.

He joined BIT as a Senior Research Fellow in 1970 and became Associate Professor in 1980. While he was with BIT, he supervised a number of research projects in the areas of microwave communications and plasma engineering. During 1983-1988, he was with the University of Dar es Salaam (UDSM), Tanzania, where he rose to the level of Professor of telecommunications with the Department of Electrical Engineering in 1986. At UDSM, he was responsible for the collaborative project "Satellite Communications for Rural Zones" with Eindhoven University of Technology, The Netherlands. From February 1988 to June 1999, he was with Telecommunications and Traffic-Control Systems Group, Delft University of Technology (DUT), The Netherlands, where he was actively involved in the area of wireless personal and multimedia communications (WPMC). He was the Head of the Transmission Research Section of IRCTR (International Research Center for Telecommunications-Transmission and Radar) and also Program Director of a newly established Center for Wireless Personal Communications (CEWPC). Since June 1999, he is with Aalborg University, Denmark as Codirector of the Center of Personal Communication (CPK) and Chair of the Wireless Information and Multimedia Group. He is currently involved in the European ACTS project FRAMES (Future Radio Wideband Multiple Access System) as a Project Leader. He is Project Leader of several international industrial funded projects. He has published more than 300 technical papers and authored two books CDMA for Wireless Personal Communications and Universal Wireless Personal Communications, and coauthored 
three books Wideband CDMA for Third Generation Mobile Communications, OFDM for Wireless Multimedia Communications, and Third Generation Mobile Communication Systems (Boston, MA: Artech House). His current research interest lies in wireless networks, packet communications, multiple access protocols, adaptive equalizers, spread-spectrum CDMA systems, and multimedia communications.

Dr. Prasad has served as a member of the advisory and program committees of several IEEE international conferences. He has also presented keynote speeches, invited papers, and tutorials on WPMC at various universities, technical institutions, and IEEE conferences. He was the Organizer and Interim Chairman of the IEEE Vehicular Technology/Communications Society Joint Chapter, Benelux Section. He is now the Elected Chairman of the joint chapter. $\mathrm{He}$ is also founder of the IEEE Symposium on Communications and Vehicular Technology (SCVT) in the Benelux and he was the Symposium Chairman of SCVT'93. He is the Coordinating Editor and Editor-in-Chief of the Kluwer International Journal on Wireless Personal Communications and also a member of the editorial board of other international journals, including the IEEE Communications Magazine and the IEE Electronics Communication Engineering Journal. He was the Technical Program Chairman of the PIMRC'94 International Symposium held in The Hague, The Netherlands, during September 19-23, 1994, and also of the Third Communication Theory Mini-Conference in conjunction with the GLOBECOM'94 held in San Francisco, CA, November 27-30, 1994. He was the Conference Chairman of IEEE Vehicular Technology Conference, VTC'99 (Fall), Amsterdam, The Netherlands, held on September 19-22, 1999. He is the General Chair of the Fourth International Symposium on Wireless Personal Multimedia Communications (WPMC'01) to be held in Aalborg, Denmark on September 9-12, 2001. He is the Founding Chairman of the European Centre of Excellence in Telecommunications, known as HERMES. He is listed in the U.S. Who's Who in the World. He is a Fellow of the IEE, a Fellow of the Institution of Electronics \& Telecommunication Engineers, and a member of The Netherlands Electronics and Radio Society (NERG). 